SCIENTIFIC LETTER

\title{
High incidence of occult femoral vein thrombosis related to multiple venous sheaths during electrophysiological studies
}

\author{
V Davutoglu, S Kervancioglu, H Dinckal, S Soydinc, S Turkmen, I Akdemir, M Aksoy
}

Heart 2004;90:1061-1062. doi: 10.1136/hrt.2003.027128

$\mathrm{T}$

here are no evidence based studies dealing with the incidence of venous sheath related thrombosis and the role of heparin, particularly in the setting of prolonged procedures with multiple lines. The clinical importance of catheter related thrombosis remains undefined in the setting of electrophysiological studies (EPS). We sought to determine the incidence of multiple venous sheath related thrombosis and the role of fixed dose, body weight independent, low molecular weight heparin (LMWH) for prophylaxis of venous thrombosis in patients undergoing EPS.

\section{METHODS}

We examined 27 consecutive patients undergoing EPS Institutional approval and informed consent were obtained. Patients were randomised into two groups according to use of LMWH (fixed dose subcutaneous injection of dalteparin 5000 units). Dalteparin was injected one hour before insertion of sheaths. All catheters (Cordis Inc, Miami, USA) were inserted using the Seldinger technique in the same side of the femoral vein. The size of the catheter sheaths ranged from 6-8 French. At catheter insertion the following parameters were recorded: patient age and catheter duration, number, position, type, and size. Investigation and diagnosis of venous thrombosis was made by ultrasound examination. After EPS was completed, the catheters were immediately removed and firm pressure was applied by hand to the femoral vein area for 15-20 minutes. Follow up ultrasound examination was performed at 24 hours, one week, and one month after catheter removal.

A diagnosis of catheter related thrombosis was based on the visualisation of an intravascular thrombosis, incompressibility of the vein by probe pressure, and absence of spontaneous flow by Doppler. Sites of deep vein thrombosis were recorded. Scans were performed with a diagnostic ultrasound system (Siemens Sonoline Elegra Advanced) using a high resolution 5.1-9.0 MHz curved linear array probe. In each patient, the uncannulated contralateral femoral vein served as a control. All examinations were recorded and reviewed by a radiologist who was unaware of the patients' state of anticoagulation and EPS procedures.

For the statistical analysis, the SPSS 10.0 version for Windows computing program was used. Categorical variables were compared by Pearson's $\chi^{2}$ or Fisher's exact test with probability values of $\mathrm{p}<0.05$ denoting significance.

\section{RESULTS}

We examined 27 consecutive patients undergoing EPS (14 women and 13 men, mean (SD) age 44 (15) years) with various diagnoses. The type, diameter, and length of catheter were standardised in order to reflect the normal clinical setting. Three femoral introducer sheaths (6, 7, and 8 French) were present in the same femoral vein in 22 patients and two femoral introducer sheaths (6 and 7 French) were present in five patients. The duration of EPS procedures from insertion to removal of sheaths was 109 (55) minutes. Eleven randomly chosen patients received prophylactic LMWH and 16 patients did not receive LMWH. In the group receiving prophylactic LMWH, only two patients developed femoral venous thrombosis on the same side of the femoral catheter. In the group not receiving LMWH, 10 patients developed femoral venous thrombosis on the same side of the femoral catheter $(\mathrm{p}=0.02)$.

There were two kinds of thrombosis: sleeve-like thrombosis, resembling the shape of a tube in the longitudinal view; and occlusive thrombosis, which appeared solid in both ultrasonic views. The shapes of the thrombosis were sleevelike in seven patients (fig lA) and occlusive in three patients. All thromboses were adherent to the venous wall at the site of venepuncture. Length of thrombosis was 1.7 (1.3) cm. None of our patients developed deep vein thrombosis in the control leg. Neither increased duration of the EPS procedure, nor number of introducer sheaths, appeared to increase the
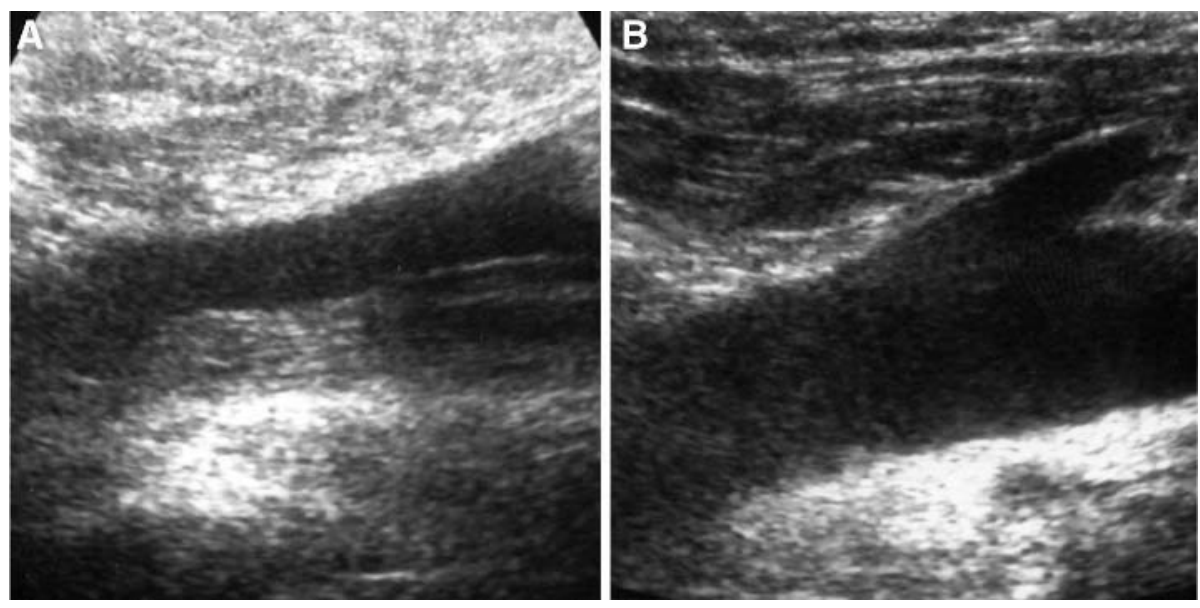

Figure 1 Ultrasound examination of femoral veins. (A) Sleeve-like thrombosis after sheath removal at 24 hours. (B) Thrombosis resolution after one week of oral anticoagulant treatment with coumadine in the same patient. 
rate of thrombosis $(p>0.05)$. Patients with deep vein thrombosis had no clinically recognisable signs. Almost all thrombosis cases responded to coumadine treatment with thrombosis resolution (fig $1 \mathrm{~B}$ ) in approximately one week to one month, except one patient who was resistant to coumadine therapy. None of our patients exhibited clinical evidence suggesting pulmonary embolism.

\section{DISCUSSION}

This prospective randomised study was the first report to demonstrate a significant increase in femoral vein thrombosis following the use of multiple femoral venous sheaths, without using heparin, in patients undergoing EPS. The incidence of femoral vein thrombosis was high (62.5\%); however, prophylactic fixed dose, body weight independent LMWH significantly decreased the risk of femoral thrombosis in these patients $(18 \%)$.

Other studies which specifically examined single iliofemoral sheath related deep vein thrombosis have reached varying conclusions regarding the increased risk from catheterisation. ${ }^{1-3}$ They concluded that physicians should be aware of the risk of the femoral route and consider ultrasound screening of the lower limb after catheter removal. A unique aspect of this study was the examination of multiple introducer sheath related thrombosis, in the same side of the femoral vein, in patients undergoing EPS. Our data clearly show that total time of the procedure did not correlate with occurrence of thrombosis, and no "safe" period for catheterisation exists. In deep vein thrombosis patients, the main risk factors may be catheter thrombogenicity, ${ }^{4}$ lack of anticoagulation, ${ }^{5}$ and occlusion of lumen secondary to multiple introducer sheaths. Increased activation of the clotting system because of ablation may be one of the additional factors for thrombosis in our patients. None of our patients experienced clinical signs of pulmonary embolism or paradoxical systemic embolism during removal of the catheter at any time before discharge from the hospital. Although we did not search for pulmonary embolism with specific tests and ruled out silent pulmonary embolism, they do not seem to pose a clinical problem if these thromboses do occur. However, all thromboses have the potential to form embolisms. In our study almost all of the thrombosis patients responded to coumadine treatment and thrombosis was resolved in approximately 1-4 weeks, except in the case of one patient who was resistant to coumadine therapy.

This study has some limitations related to the small number of patients; nevertheless, the difference in the incidence of thrombosis formation in the group of patients not receiving LMWH was nearly 3.5 times higher than that in the group receiving LMWH.

In conclusion, our study demonstrates a high incidence of occult deep vein thrombosis during uncomplicated routine multiple femoral venous catheterisation in patients undergoing EPS. Prophylactic fixed dose, body weight independent LMWH significantly reduces the incidence of thrombosis formation relating to multiple femoral vein sheaths.

\section{Authors' affiliations}

V Davutoglu, H Dinckal, S Soydinc, S Turkmen, I Akdemir, M Aksoy, Department of Cardiology, University of Gaziantep, Sahinbey Medical Center, Gaziantep, Turkey

S Kervancioglu, Department of Radiology, University of Gaziantep

Correspondence to: Dr Vedat Davutoglu, Guneykent mah, Besyuzevler sitesi 7.Blok, Daire 10. 27310 Sahinbey, Gaziantep, Turkey;

vedatdavutoglu@hotmail.com

Accepted 22 December 2003

\section{REFERENCES}

1 Durbec $\mathrm{O}$, Viviand $\mathrm{X}$, Potie $\mathrm{F}$, et al. Lower extremity deep vein thrombosis: a prospective randomized, controlled trial in comatose or sedated patients undergoing femoral vein catheterization. Crit Care Med 1997;25:1982-5.

2 Trottier SJ, Veremakis C, O'Brien J, et al. Femoral deep vein thrombosis associated with central venous catheterization: results from a prospective randomized trial. Crit Care Med 1995;23:52-9.

3 Meredith JW, Young JS, O'Neil EA, et al. Femoral catheters and deep venous thrombosis: a prospective evaluation with venous duplex sonography. J Trauma 1993;35:187-91.

4 Stillman RM, Soliman F, Garcia L, et al. Etiology of catheter-related sepsis, correlation with thrombogenicity. Arch Surg 1977;112:1497-9.

5 Bern MM, Lokich JJ, Wallach SR, et al. Very low dose of warfarin can prevent thrombosis in central venous catheters: a randomized prospective trial. Ann Intern Med 1990;1 12:423-8.

\section{IMAGES IN CARDIOLOGY}

\section{Acupuncture for chest pain}

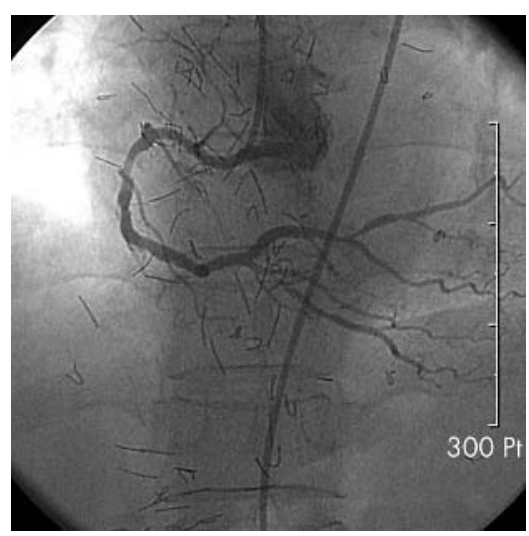

72 year old woman presented with a five year history of anterior chest pain. She had a medical history of hypertension, osteoarthritis, and tension headache. Her chest pain was substernal in location, manifested as a burning sensation, and was aggravated by exercise. Her ECG showed T wave inversion in leads II, III, aVF, and V4-V6. A right coronary angiogram revealed diffuse wall irregularities over the middle right coronary artery; incidentally, numerous metallic shadows of acupuncture in the chest wall were also observed. She had been treated many times with acupuncture for her chest pain, reflecting the fact that many Asian elderly people depend on treatment with acupuncture needles for pain control. 